\title{
Instrumentos y trabajos geodésicos de la Armada: \\ la Comisión Hidrográfica de la Península y el Observatorio de San Fernando (1859-1887)
}

\author{
F. JOSÉ GONZÁlEZ GONZÁLEZ \\ M. BERROCOSO DOMÍNGUEZ
}

\section{RESUMEN}

Este artículo presenta un breve estudio sobre los trabajos geodésicos emprendidos por la Armada Española en el siglo XIX, y los instrumentos utilizados por el Observatorio de San Fernando y por las comisiones hidrográficas, especialmente la Comisión Hidrográfica de la Península, encargada del levantamiento cartográfico de las costas españolas.

\begin{abstract}
This article introduces a brief survey on the geodetic tasks carried out by the Spanish Navy in the 19th century, and the scientific instruments used by the San Fernando Observatory and the hydrographical expeditions. Special attention deserves the Iberian Peninsula Hydrographical Expedition, in charge of surveying the Spanish coasts.
\end{abstract}




\section{El Observatorio de la Marina y la Hidrografía}

La recuperación naval de la España de la Ilustracion, caracterizada, entre otras cosas, por el fomento de la navegación y de la hidrografía, trajo como consecuencia el aumento de los trabajos cartográficos emprendidos por oficiales de la Armada, tanto en las costas de la Península como en América y otros territorios de ultramar. En todos ellos, la colaboración del Real Observatorio de Cádiz, fundado por Jorge Juan en 1753, fue esencial y se produjo en dos vertientes. Por un lado, mediante la participación en dichos trabajos de su propio personal o de oficiales con preparación científica adquirida en el llamado curso de estudios mayores o sublimes, que en él se impartía. Por otro lado, mediante el préstamo de instrumentos de la institución a las expediciones hidrográficas o la supervisión de la adquisición de los aparatos necesarios no disponibles en el propio Observatorio ${ }^{1}$. Expediciones como las de Tofiño (1783-1789) para el levantamiento de las costas españolas, Antonio de Córdoba (1785) y Cosme de Churruca (1788) al estrecho de Magallanes, Alejandro Malaspina alrededor del mundo (1789-1794), Cosme de Churruca a Trinidad y las Antillas (1792) o Joaquín Francisco Fidalgo a las Costas de Colombia y Venezuela (1796) contaron con el apoyo del observatorio gaditano, tanto en los aspectos relacionados con el personal como en la dotación de instrumentos científicos ${ }^{2}$.

Además, desde 1770, el Depósito Hidrográfico de Madrid, estuvo encargado de grabar y publicar las cartas marítimas, de redactar los derroteros y de coordinar las comisiones hidrográficas organizadas por la Armada. Más adelante, la necesidad de conservar, reproducir y publicar los importantes trabajos desarrollados por la generación de marinos científicos encabezada por Tofiño, que realizaron numerosos y precisos levantamientos hidrográficos de las costas españolas y americanas, trajo consigo la creación, en 1797, de la Dirección de Hidrografía, entre cuyos objetivos estaban, además de los que antes habían ocupado al Depósito, la formación de personal especializado en el grabado de cartas, la centralización de todo lo relacionado con la hidrografía y el fomento del estudio de las técnicas hidrográficas entre el personal de la Armada. La dirección de esta nueva dependencia fue desempeñada, a partir de entonces, por importantes personajes en la historia de la Marina española: José de Espinosa y Tello (1797-1802), Juan Gutiérrez de la

\footnotetext{
1 Sobre la historia del Observatorio de la Marina durante los siglos XVIII y XIX véanse las obras de Lafuente, A. y Sellés, M. (1988): El Observatorio de Cadiz (1753-1831), Madrid; y GonzÁLEZ, F.J. (1992): El Observatorio de San Fernando (1831-1924), Madrid.

2 Los trabajos cartográficos llevados a cabo en América por marinos españoles han sido estudiados recientemente por Rivera Novo, B. y MARTín-MERÁs, L. (1992): Cuatro siglos de cartografía en América, Madrid.
} 
Concha (1802-1803), Joaquín Francisco Fidalgo (1810) o Felipe Bauzá (1810$1822)^{3}$

A principios del siglo XIX, mientras el Observatorio intentaba organizar sus trabajos en su nuevo emplazamiento de la Isla de León, el fructífero período vivido por la hidrografía española sufrió los bruscos cambios que afectaron a la Marina, para la que Trafalgar no hizo más que confirmar la existencia de una profunda crisis iniciada algunos años atrás. A la precaria situación de la Armada habría que añadir, sin duda, los efectos desastrosos de la guerra contra los franceses. La Dirección de Hidrografía, dependiente del Gobierno y ubicada en la capital del Reino, hubo de sufrir constantes vicisitudes, tanto en la guerra como en los años de confusión política que la siguieron. Sus trabajos fueron paralizados en diversas ocasiones y gran parte de su personal tuvo que optar por el exilio. Todo ello afectó directamente al trabajo científico organizado por la institución y a la continuidad de sus publicaciones.

No obstante, a pesar de la catastrófica situación de la Marina en los primeros años del XIX, la actividad de la Dirección de Hidrografía no cesó, siendo muchos los proyectos e iniciativas presentados a las autoridades por su director, Felipe Bauzá. La figura de Bauzá está detrás de asuntos tan diversos e interesantes como el proyecto de creación de un Cuerpo de Ingenieros Hidrógrafos (1816), el proyecto para la elaboración de una Carta Geográfica General de España (1820), la publicación del Derrotero de las Antillas (1820) o los trabajos para la terminación de los Atlas de América del Sur y del Norte (terminados en 1828 y 1830 , respectivamente) ${ }^{4}$.

Su sucesor fue Martín Fernández de Navarrete que estuvo al frente de la institución hasta 1844. Prolífico escritor e historiador de los hechos relacionados con la Marina, Fernández Navarrete dirigió los trabajos hidrográficos en un período de profunda decadencia. La Marina atravesaba uno de sus peores momentos y el país, sumido en las convulsiones políticas y en la guerra carlista, no estaba mucho mejor. De todas formas, en una época en la que era normal el alquiler de barcos y tripulaciones a otros países para determinadas campañas, consiguió mantener la actividad de comisiones hidrográficas en Filipinas y las Antillas, aunque en la Península había que seguir utilizando las cartas levantadas por Tofiño en el siglo XVIII, pues desde entonces no había funcionado en nuestras costas ninguna comisión hidrográfica ${ }^{5}$.

\footnotetext{
${ }^{3}$ El proceso de creación de las instituciones cartográficas de la Armada ha sido estudiado por Martín-Merás, L. y Rivera, B. (1990): en el estudio que precede al Catálogo de cartográfica histórica de España del Museo Naval, Madrid.

${ }^{4}$ La actividad de Felipe Bauzá ha sido estudiada por Martín-Merás, L. (1984) en su artículo: «Felipe Bauzá: Sus trabajos sobre el Mapa de España», Revista de Historia Naval, 20, 107-112.

5 Véase GonZÁlez, F.J. (1992), p. 274.
} 
La adquisición, por parte de la Marina, de los instrumentos necesarios para la ejecución de los trabajos hidrográficos encomendados a las comisiones destinadas al efecto empezó a ser habitual durante la segunda mitad del siglo XVIII. Estos instrumentos, antes de pasar a su destino, debían ser inspeccionados en el Observatorio, donde se procedía a su anotación en el inventario ${ }^{6}$. Sin embargo, la crisis de los primeros años del siglo XIX dejó notar pronto sus efectos. Como consecuencia, la adquisición oficial de instrumentos cesó durante un largo período de tiempo y los oficiales de la Armada, que tenían serias dificultades para cobrar sus sueldos, no quedaron precisamente en una situación muy favorable para la adquisición de sus propios instrumentos. Este estado de cosas se prolongó durante bastante tiempo, dando lugar a la paradójica situación de que, hacia 1830, los escasos buques operativos de la Armada contasen con una peor dotación instrumental que la que había sido normal treinta años antes?

En 1844, fue ordenada la creación de un depósito de instrumentos en el Observatorio de San Fernando. Unos años después, el nuevo reglamento de la institución, aprobado en 1859, recogió de forma explícita la misión del Observatorio como depósito de instrumentos de la Marina. A partir de entonces, este establecimiento contaría con una sección dedicada especialmente a los instrumentos de la marina militar. Según el mencionado reglamento ${ }^{8}$, las funciones de la nueva sección, conocida como Sección de Geografía, serían las siguientes:

"En la quinta sección, que se denominará «de geografia», radicarán todos los asuntos relativos a las aplicaciones de la fisica y de la astronomia, a la geografia y navegación; y en tal concepto en ella se custodiarán, conservarán y arreglarán los cronómetros de la marina militar, los instrumentos de fisica, de astronomía náutica y de geodesia, destinados al servicio de la Armada, y las colecciones de atlas, cartas, portulanos, derroteros e instrucciones que hayan de emplearse en las necesidades del servicio; se cuidará que las existencias de cronómetros, instrumentos de todas clases, atlas, portulanos, derroteros, etc., sean proporcionadas a las necesidades probables del mismo; se sostendrá y conservará archivada la correspondencia a que den lugar las adquisiciones y entregas de cronómetros, instrumentos, etc.»

\footnotetext{
${ }^{6}$ El primero de estos inventarios, que actualmente se conserva en la Biblioteca de Real Observatorio de la Armada (San Fernando) se titula Inventario de los instrumentos pertenecientes al Observatorio Real de Cádiz, y sus anotaciones comienzan el 11-2-1789.

${ }^{7}$ La instrumentación científica de la Armada durante los siglos XVIII y XIX ha sido objeto de estudio en un proyecto de investigación, auspiciado por el Instituto de Historia y Cultura Naval y desarrollado por los autores de este artículo durante los años 1992 y 1993. Los resultados de este trabajo están actualmente en prensa.

${ }^{8}$ Reglamento para el régimen, dirección y gobierno del Observatorio de Marina de San Fernando (1959), Madrid.
} 


\section{INSTRUMENTOS Y TRABAJOS GEODESICOS DE LA ARMADA}

La entrada en vigor del reglamento de 1859 vino acompañada de una nueva disposición oficial, en la que se regulaba todo lo relativo a la adquisición y arreglo de instrumentos por particulares o instituciones que no fuesen el Observatorio de San Fernando. Quedó establecido entonces que todos los instrumentos y cronómetros de la Marina tendrían que ser adquiridos por la dirección del Observatorio de San Fernando, exceptuando aquellos casos que requiriesen una especial urgencia o que contasen con autorización expresa del Gobierno?.

A partir de entonces, la relación entre el Observatorio y la hidrografía quedaría reflejada en sucesivas disposiciones oficiales. En 1873, un nuevo reglamento incluyó entre los trabajos asignados al Observatorio de San Fernando la ejecución de las observaciones astronómicas conducentes a la rectificación de las posiciones hidrográficas de las costas de la Península y posesiones de ultramar, que debían ser realizadas en combinación con la comisiones hidrográficas ${ }^{10}$. Unos años más tarde, la reorganización de la Armada, llevada a cabo en 1908, suprimió la Dirección de Hidrografía, organismo que había regido la hidrografía en la Armada durante más de un siglo. La misma disposición oficial que la hizo desaparecer estableció que el Estado Mayor Central debía encargarse de planificar los trabajos de la Comisión Hidrográfica, que la Dirección General de Navegación y Pesca tendría a su cargo el grabado y publicación de los planos levantados por la citada comisión y que del Observatorio de San Fernando saldrían las instrucciones referentes a los métodos que se debían utilizar en los cálculos astronómicos, geodésicos y magnéti$\cos ^{11}$.

Desde 1924 el Observatorio se regiría por un nuevo reglamento que, entre las misiones encomendadas a la institución, estipuladas en el primero de sus artículos, incluía la práctica y reducción de todas las observaciones y experiencias físicas que pudiesen contribuir a los adelantos de la navegación y de la hidrografía y el establecimiento de fórmulas y métodos para la parte cientifica en los trabajos hidrográficos que se ejecutasen. Poco después, en 1927, fue creado el Servicio Hidrográfico de la Armada, que comenzaría su andadura como una sección del Observatorio de San Fernando. De esta forma, el director del Observatorio pasó a ser también director de Hidrografía, iniciándose así un período de actividad hidrográfica en el Observatorio que se prolongó hasta 1944, fecha de creación del actual Instituto Hidrográfico de la Marina en Cádiz ${ }^{12}$.

\footnotetext{
9 Real Orden de 27-12-1859. Véase Colección Legislativa de la Armada (1909), Madrid.

10 "Reglamento del Instituto y Observatorio de Marina», aprobado el 29-5-1873 y publicado en Estado General de la Armada para el año 1875 (1874), Madrid.

11 Real Orden de 16-1-1908, véase GonZÁLEZ, F.J. (1992), p. 277.

12 Véase GonZÁLEZ, F.J. (1992), p. 278.
} 


\section{F. JOSE GONZALEZ GONZALEZ Y M. BERROCOSO DOMINGUEZ}

\section{Geodesia y cartografía en la España del siglo XIX}

Las necesidades cartográficas demandadas por la sociedad de los siglos XVIII y XIX, tanto a nivel militar (topografía) como a nivel civil (catastro, obras públicas) provocaron un importante desarrollo de una nueva ciencia, la geodesia. Como consecuencia, en el siglo XIX la cartografía se convirtió en una herramienta fundamental para el desarrollo teórico y práctico de la geodesia, influyendo decisivamente en un avance espectacular en cuanto a instrumentación y exactitud de los resultados cartográficos. Si bien inicialmente las diferencias entre geodesia y cartografía eran prácticamente nulas, con el paso del tiempo se fue consolidando la geodesia como una ciencia dotada de mayores precisiones y exactitudes en las mediciones efectuadas, mientras que la cartografía fue adquiriendo una utilidad práctica que no requería de tal grado de precisión ${ }^{13}$.

Para alcanzar unos óptimos resultados, fue imprescindible una mejora en la construcción de los instrumentos de medida, tanto de las bases geodésicas (reglas) como de las direcciones angulares (teodolitos). El perfeccionamiento en la construcción de teodolitos se materializó en la aplicación de varios microscopios para realizar las lecturas en los círculos graduados y en la obtención de la lectura media de todos ellos. Además, se utilizó el método de la inversión de círculos (derecha, izquierda) para la obtención de cada medida. Las complicaciones en la construcción de aparatos para medir bases fueron bastante más numerosas, pues no sólo había que tener en cuenta las características especiales de precisión en la lectura de las medidas. Cuestiones como el manejo del aparato o la rentabilidad económica de la distancia medida en función del potencial humano necesario y del tiempo empleado tuvieron también su importancia ${ }^{14}$.

En el aspecto técnico, la construcción de una regla de medir bases fue acompañada de la búsqueda de materiales sobre los que las variaciones termométricas e higrométricas fuesen mínimas. Así, se diseñaron reglas bimetálicas de platino y latón (Borda, 1798), de hierro forjado (Tenner, 1820; Struve, 1827), de hierro forjado y latón (Colby, 1827; Porro, 1850), de hierro forjado y zinc (Bessel,1834). Las principales diferencias entre estas reglas, diseñadas usualmente en varios cuerpos (de aproximadamente cuatro metros cada uno), radicaban en los ingeniosos métodos utilizados para disminuir o controlar la dilatación de los distintos materiales entre cuerpo y cuerpo.

\footnotetext{
13 Sobre la evolución de la geodesia a partir del siglo XVIII, véase LAFUENTE, A. y Delgado, A. J. (1984): La geometrización de la Tierra (1735-1744), Madrid.

14 Véase Berrocoso, M. y GonZález, F.J. (1991): «La geodesia en el Observatorio de San Fernando durante la segunda mitad de siglo XIX", Actas del V Congreso de la Sociedad Española de Historia de las Ciencias y de las Técnicas, tomo II, Murcia.
} 
Aunque el hundimiento general del movimiento ilustrado paralizaría la mayoría de las actividades científicas en nuestro país, todavía quedaba un ambiente favorable a los trabajos geográficos a principios del siglo XIX. Sin embargo, la Guerra de la Independencia acabaría con todas las posibilidades de desarrollar un programa cartográfico parecido a los que se llevaron a cabo en otros países europeos. De ahí que, entre 1808 y 1814, tanto franceses como ingleses tuviesen que desarrollar importantes trabajos cartográficos y topográficos, ante la inexistencia de unos mapas fiables de los territorios por los que se llevaban a cabo las campañas de la guerra ${ }^{15}$.

Tras la muerte de Fernando VII se produjo un cierto resurgir de los estudios geográficos con los trabajos de Madoz y Coello. En 1836 fue creado definitivamente el Cuerpo de Estado Mayor, cuyos oficiales recibían una buena formación en topografía y geodesia, y dos años más tarde fue establecido el Depósito de la Guerra, encargado de las secciones cartográficas del Estado Mayor. Después del intento frustrado de crear una Escuela de Ingenieros Geógrafos bajo la inspección del Observatorio de Madrid (1835), fue planteada en 1840 la necesidad de llevar a cabo un proyecto de Mapa de España. Por primera vez desde 1820, año en que las Cortes y Felipe Bauzá habían recomendado algo parecido, se producía un intento oficial de impulsar la elaboración de un mapa general del territorio nacional. Fue creada una Comisión Facultativa encargada de los trabajos y regulada la adquisición de los instrumentos; sin embargo, tras varias reorganizaciones, la citada comisión no fue capaz de llevar adelante el trabajo encomendado y la situación volvió a ser la misma que al principio.

Conforme pasaban los años, la necesidad de abordar la triangulación geodésica y elaborar un mapa se fue haciendo más importante. En 1853 fue creada, dependiente del Ministerio de Fomento, la Dirección de la Carta Geográfica de España. Unos meses más tarde, se estableció la formación del mapa por personal del Ministerio de la Guerra. De esta forma, en 1854, una comisión formada por jefes y oficiales del Ejército (cuerpos de Artillería, Ingenieros y Estado Mayor) emprendería los trabajos para el establecimiento de una red geodésica de carácter nacional. Fueron éstos los años del diseño y construcción del llamado aparato de Ibáñez. Este aparato estaba dotado de micrómetros de hilos móviles y otras disposiciones análogas a las de los anteojos de los instrumentos de astronomía. Su utilización por la Comisión del Mapa de España supuso una verdadera revolución dentro del campo de la geodesia, debido a las grandes precisiones que con él se conseguían ${ }^{16}$.

\footnotetext{
15 Véase NúÑEZ dE LAS Cuevas, R. (1982): «Cartografía española en el siglo XIX», Historia de la cartografía española, Madrid.

${ }^{16}$ El aparato de Ibáñez está descrito en Alvarez Sereix, R. y Bellón de Arcos, J. (1889): Aparato de Ibáñez para medir bases geodésicas, Madrid.
} 
El volumen adquirido por los datos obtenidos y la importancia específicá de proyecto, influyeron decisivamente en la creación de una institución cartográfica, el Instituto Geográfico, y en el nombramiento del Coronel Ibáñez e Ibáñez de Ibero, como primer director. A partir de entonces, la nueva institución civil sustituiría al Depósito de la Guerra en todo lo relacionado con los trabajos sobre la determinación de la forma y dimensiones de la Tierra, las triangulaciones geodésicas y topográficas, las nivelaciones de precisión y los asuntos de catastro, pesas y medidas. No obstante, esto no significó una ruptura en la continuidad de los trabajos geodésicos, realizados hasta entonces por los militares y por el Depósito de la Guerra, pues no hay que olvidar que hasta finales del siglo XIX los militares ocuparon importantes cargos directivos en el nuevo Instituto Geográfico ${ }^{17}$.

La tarea primordial del Instituto dirigido por Ibáñez, sería la elaboración del Mapa Topográfico Nacional a escala 1/50.000, cuya primera hoja salió publicada en 1875 (Madrid-559). No obstante, para su realización era necesario completar la red geodésica iniciada en 1858 , de ahí que la mayor parte de los trabajos del Instituto durante sus primeros tiempos fueran de carácter geodésico, alcanzándose pronto un nivel destacado respecto a Europa. A la precisa medida de la base central de la tringulación en Madridejos, habría que añadir las iniciativas de Ibáñez en lo que se refiere a la construción de una regla de gran precisión y sencillez, el ya citado aparato de Ibáñez, usada tanto en España como en la medida de diversas bases geodésicas extranjeras. Entre los trabajos más importantes llevados a cabo por Ibáñez, una de las figuras científicas más destacadas de la España del XIX, no podemos olvidar el enlace de la red geodésica española con la de Argelia, llevado a cabo en colaboración con Francia en 1878.

\section{La Comisión Hidrográfica de la Península}

Mientras tanto, la Armada no quedó atrás en lo que se refiere al impulso de los trabajos geodésicos y cartográficos. A mediados del XIX, el jefe de la Dirección de Hidrografía, Joaquín Gutiérrez de Rubalcaba, promovió una reorganización de los trabajos hidrográficos, que culminaría unos años después con la creación de tres comisiones hidrográficas, las correspondientes a la Península, las Antillas y las Filipinas ${ }^{18}$. Por otro lado, en 1856 dió comien-

17 Véase GonZález, F.J. (1992): p. 272.

18 Sobre la creación de las comisiones hidrográficas de la Armada española a mediados del siglo XIX, véase la obra ya citada de MARTín-MERÁs, L. y RiVERA, B. (1990) y el artículo de Rivera Novo, B. (1984) : "Renovación científica de la hidrografía española en el siglo XIX", Revista de Historia Naval, 7. 
zo en el Observatorio de San Fernando el primer Curso de Estudios Superiores. Este curso estaba orientado hacia la formación de una serie de oficiales de Marina que, sin abandonar totalmente su profesión, debían dedicarse a adquirir los conocimientos teóricos (matemáticas, física, idiomas) y prácticos (astronomía, geodesia) considerados necesarios por la Armada para acceder a ciertos destinos cualificados (Observatorio, Depósito Hidrográfico, tareas docentes del Colegio Naval y comisiones científicas e hidrográficas) ${ }^{19}$.

A finales de 1860, el vapor Piles fue designado como buque encargado de los trabajos hidrográficos desarrollados por la Marina en las costas peninsulares. A este buque fueron destinados los oficiales procedentes de la primera promoción del Curso de Estudios Superiores, citado anteriormente. Además del comandante de la Comisión, teniente de navío Manuel Fernández Coria, embarcaron en el Piles como oficiales José Montojo, Agustín Serrano, Cecilio Pujazón y Simón Manzano. Unos meses más tarde, por Real Orden de 12 de octubre de 1861, fue aprobado el «Proyecto de instrucciones generales para el levantamiento de las costas de la Península e islas adyacentes", que había sido elaborado por la Dirección de Hidrografía, con la intención de que pudiesen enlazar tanto con los trabajos de la Comisión de la Carta de España como con las redes geodésicas de Portugal y Francia ${ }^{20}$. Este proyecto marcaba los siguientes objetivos a la comisión hidrográfica embarcada en el vapor Piles:

- Medir una base con exactitud para apoyar sobre ella una cadena de triángulos geodésicos de primer orden, extensiva a Portugal y Francia.

- Establecer, a partir de esta red, triángulos de $2 .^{\circ}$ y $3 .^{\text {er }}$ orden como base para los trabajos topográficos e hidrográficos de detalle.

- Determinar las alturas de los vértices sobre el nivel del mar (tomando distancias cenitales recíprocas).

- Determinar las posiciones geográficas de los vértices de los triángulos principales que correspondiesen a puntos principales de la costa.

- Llevar un diario meteorológico completo, observar las mareas y estudiar la declinación y la inclinación magnéticas.

- Levantar hidrográficamente la costa a escala 1/100.000 (1/50.000 cuando lo exigiese la abundancia de detalles).

- Levantar con detalle los planos de los puertos y de los ríos navegables.

19 Véase GonZÁLEZ, F.J. (1988) : «El Real Observatorio de la Armada y su faceta docente: Los estudios superiores (siglos XVII y XIX)", Gades, 18, 65-85, Cádiz.

20 Véase el «Extracto de la memoria redactada por el capitán de fragata don Rafael Pardo de Figueroa, jefe de la Comision Hidrográfica de la Península, acerca de los trabajos verificados por ésta desde 1876 a 1887", Anuario de la Dirección de Hidrografía (1888), Madrid. 
- Sondear la costa hasta los 167 m. (100 brazas) y determinar la posición de los principales accidentes (bajos, arrecifes).

- Elaborar memorias descriptivas con vistas de la costa e informes de las operaciones de cálculo y observación realizadas, con descripción de los instrumentos utilizados.

Durante los primeros años de funcionamiento de la Comisión, aquellos en los que estuvo al mando de la misma el teniente de navío Fernández Coria (1860-1864), los trabajos se fueron desarrollando con lentitud, debido principalmente a la inexperiencia del personal y a la escasa dotación instrumental embarcada en el vapor Piles.

Entre 1864 y 1876 la jefatura de la Comision fue desempeñada por José Montojo y Salcedo. Durante estos años, el desarrollo de los trabajos hidrográficos se vió afectado por la inestabilidad política del momento (pronunciamiento de 1868, guerra carlista, insurrección cantonalista de 1873). No obstante, la llegada de algunos de los instrumentos encargados a la casa Brunner en París, de los que trataremos con detenimiento más adelante, y la asignación de dos buques auxiliares a las tareas hidrográficas (el falucho Caimán y el vapor auxiliar Relámpago), consiguieron influir decisivamente en la obtención de resultados bastante positivos (Véase el Apéndice I).

Tras esta primera etapa, fue nombrado jefe de la Comision Hidrográfica Rafael Pardo de Figueroa, que estuvo al frente de la misma hasta 1887. Durante estos años, además de ser finalizados los trabajos en la costa mediterránea hasta la frontera con Francia, se iniciaron los cálculos y correcciones de todo lo realizado hasta entonces. Fueron once años en los que la Comisión mejoró ostensiblemente la calidad y la fiabilidad de los resultados, gracias a la adquisición de nuevos instrumentos, a la aplicacion de las fórmulas esféricas de Hausen para determinar posiciones geográficas y a la adición de una segunda corrección a los resultados obtenidos (Véase el Apéndice II).

En 1887, el capitán de fragata José Gómez Imaz sustituyó a Pardo de Figueroa al frente de la Comisión. Nada más empezar los trabajos pendientes en la costa norte de Cataluña, el vapor Piles tuvo que ser sustituido por un nuevo buque, el Vulcano, con el que se terminarían los trabajos en la costa mediterránea durante los años 1888 y 1889. El siguiente objetivo de la Comsión Hidrográfica fue el archipiélago balear, en el que los trabajos avanzarían a buen ritmo gracias al sistema empleado, consistente en que dos equipos trabajasen simultáneamente, uno haciendo el levantamiento de la costa y otro el de los puertos. La hidrografía de las islas Baleares no se terminaría hasta unos años después, siendo comandante de la Comisión Emilio Luanco y Gaviot. Durante su período al frente de la misma, la Comisión se trasladó al Cantábrico, una vez terminados todos los 


\section{INSTRUMENTOS Y TRABAJOS GEODESICOS DE LA ARMADA}

levantamientos en el Mediterráneo, y el Vulcano fue sustituido por un nuevo buque, el Urania.2.

\section{Las colecciones de instrumentos geodésicos de la Marina}

Unos meses antes de la organización de la Comisión Hidrográfica de la Península, en 1859, había sido ordenada la adquisición de unas colecciones de instrumentos geodésicos, destinadas a los trabajos de levantamientos cartográficos que debían llevar a cabo las comisiones hidrográficas ${ }^{22}$. El director del Observatorio, comisionado entonces para proceder a la adquisición de los mencionados instrumentos, recabó de la casa francesa Brunner información sobre el importe y características de los aparatos que debían componer las citadas colecciones. Con esta información, las autoridades de Marina dispusieron definitivamente la adquisición de un aparato de medir bases y tres colecciones de instrumentos geodésicos. El teniente de navío Antonio de Tomaseti fue designado por la Marina para viajar a París, con objeto de activar los trabajos de Brunner y auxiliar al artista en los trabajos necesarios para comparar la regla del aparato de medir bases con el metro patrón, conservado en el Observatorio de París ${ }^{23}$. Desde entonces, estuvo comisionado en la capital francesa donde, bajo la supervisión del director del Observatorio, procedió al encargo y adquisición de numerosos instrumentos a la conocida casa constructora de instrumental científico Brunner. Durante estos años tuvo lugar la adquisición de dos colecciones completas de instrumentos geodésicos, destinadas a las comisiones hidrográficas de la Península y de las Antillas (la Comisión de Filipinas iniciaría sus trabajos unos años más tarde), y de los instrumentos magistrales que serían instalados en el Observatorio de San Fernando ${ }^{24}$.

\footnotetext{
${ }^{21}$ Véase González, F.J. (1992), p. 277.

22 La dotación instrumental de la Comisión Hidrográfica de la Península ha sido tratada recientemente por BERRoCoso, M. y GonZÁLEZ, F.J. en «Los instrumentos científicos de la Marina española: El Real Observatorio de la Armada y la dotación instrumental de las expediciones ilustradas y de las comisiones hidrográficas», comunicación presentada en el XIXth International Congress of History of Science, celebrado en Zaragoza (1993).

${ }^{23} \mathrm{El}$ proceso de adquisición de estos instrumentos puede ser estudiado en el expediente titulado “Colecciones completas de instrumentos geodésicos para el levantamiento de planos (1859-1862)», que se conserva en el Archivo-Museo Don Alvaro de Bazán (Viso del Marqués, Cuidad Real), serie Depósito Hidrográfico, Asuntos Particulares, Leg, 4929.

${ }^{24}$ Para obtener la información referida al uso de instrumentos para las comisiones hidrográficas pueden ser utilizados los inventarios que actualmente se conservan en la Biblioteca del Real Observatorio de la Armada, especialmente los titulados: Inventario de instrumentos de geografía geométrica (1859-1864), Inventario de instrumentos y cronómetros destinados a las comisiones geodésicas e hidrográficas (1865-1867) y, por ultimo, algunos volúmenes del Historial de los instrumentos y cronómetros que posee el Estado para el Servicio del
} 
Según el contrato firmado en París por Brunner, este se comprometió a construir para la Marina española una colección magistral de instrumentos geodésicos compuesta por un aparato magistral de medir bases, similar al que ya había construido para la Comisión de la Carta de España (aparato de Ibáñez), un teodolito de primer orden, un nivel stadia con círculo horizontal y polímetro, un examinador de niveles, y una pantómetra con anteojo y nivel. Además, Brunner debía entregar a la Marina otras dos colecciones formadas cada una por un aparato secundario de medir bases, un teodolito con círculo repetidor horizontal y círculo vertical, con un anteojo de $35 \mathrm{~cm}$. de distancia focal, un nivel stadia con círculo horizontal y polímetro, una pantómetra con anteojo y nivel, una plancheta, un anteojo para medir distancias y una escala de cobre de $40 \mathrm{~cm}$. de longitud ${ }^{25}$.

En agosto de 1862 llegaron a Cádiz el aparato magistral de medir bases y el comparador construidos en París por la casa Brunner. Aunque la adquisición de estos instrumentos había sido propuesta por la Dirección de Hidrografía de Madrid, empeñada en elevar la cartografía maritima española a un nivel semejante al de los trabajos geodésicos realizados por la Comisión encargada del Mapa de España, los responsables de la Armada se decidieron por su instalación en los locales del Observatorio de San Fernando. Esta institución que, como ya hemos visto, había estado intimamente ligada a los trabajos cartográficos de la Marina desde su creación en el siglo XVIII, disponía de unos salones de observaciones, de reciente construcción, que aparecían como la ubicación idónea para estos aparatos de precisión que, para asegurar la invariabilidad de su posición una vez instalados, necesitaban unas especiales condiciones de aislamiento y cimentación. El mismo oficial que había supervisado en París la construcción de los aparatos, el teniente de navío Tomaseti, fue encargado de la dirección de las obras necesarias para su instalación en el salón occidental de observaciones.

Concluiremos este capítulo, dedicado a los aparatos geodésicos de la Armada en el siglo XIX, con la descripción de los instrumentos geodésicos magistrales, que fueron instalados en el Observatorio de San Fernando, y los aparatos secundarios de medir bases, destinados a los trabajos de las comisiones hidrográficas ${ }^{26}$.

Instituto, buque de guerra, comisiones hidrográficas y demás establecimientos oficiales de la Marina (1874-1910).

${ }^{25}$ El contrato firmado entre Tomaseti y Brunner se encuentra en el ya citado expediente "Colecciones completas de instrumentos geodésicos para el levantamiento de planos (18591862)", Archivo-Museo Don Alvaro de Bazán (Viso del Marqués, Cuidad Real), serie Depósito Hidrográfico, Asuntos Particulares, Leg, 4929.

26 Para la descripcion de estos instrumentos hemos utilizado el trabajo de BERrocoso, $\mathbf{M}$. y GonZáleZ, F. J. (1991): «La geodesia en el Observatorio de San Fernando durante la segunda mitad del siglo XIX». 


\section{Aparato magistral de medir bases}

A grandes rasgos, el aparato magistral para medir bases estaba compuesto por la regla bimetálica, los soportes, los microscopios y anteojos y los instrumentos accesorios. El núcleo del aparato era la regla bimetálica, formada por una regla de platino de $411 \mathrm{~cm}$. de largo, $2,25 \mathrm{~cm}$. de ancho y $0,5 \mathrm{~cm}$. de espesor, unida a otra regla de latón mediante dos piezas de platino que encajaban en dos aberturas existentes cerca de los extremos. Gracias a este dispositivo, las dos reglas se podían dilatar libremente y la posición relativa de la pieza de platino unida a la regla de platino y a la regla de latón indicaba las diferencias de dilatación de ambas reglas.

Las dos reglas estaban colocadas sobre una barra de hierro en forma de $\mathrm{T}$ invertida. Para que las dilataciones de esta barra de hierro no influyesen en la regla bimetálica, ésta llevaba en su parte superior 14 piezas de latón fijadas a la barra por dos tornillos cada una de ellas. Asímismo, disponía de un conjunto de niveles fijos para garantizar la horizontalidad de la barra de hierro y otro de niveles móviles para lograr la horizontalidad de las reglas de platino y de latón, una vez establecida la primera. El aparato principal de medir bases contaba, además, con cuatro soportes. Dos de ellos para el apoyo de la regla. Los otros dos sólo se utilizan en el campo para preparar la medida del tramo siguiente.

Para la lectura de las divisiones de la regla, el aparato magistral de medir bases estaba provisto de dos microscopios de 60 aumentos, que se colocaban sobre la escala de la regla. A ello habría que añadir dos anteojos, uno para observar las referencias colocadas en el suelo y otro para alinear los soportes donde se colocan los microscopios. Para marcar las referencias en el campo se utilizaba un aparato con una cruz grabada en metal, que es la que se visaba con uno de los anteojos anteriormente citados. También se utilizaban unos pies de madera para colocar a la altura conveniente los soportes descritos más arriba.

\section{Comparador Brunner}

El comparador Brunner estaba formado por un muro de piedra que soportaba cuatro microscopios y que se prolongaba por debajo del piso de madera del salón donde fue instalado, de forma que el movimiento de los observadores no afectaba a las medidas efectuadas. Este dispositivo fue ideado para comparar los aparatos secundarios con el aparato principal, antes y después de cada utilización práctica. El comparador Brunner disponía de una serie de aparatos de apoyo para verificar con toda precisión los mencionados análisis: cuatro microscopios micrométricos, un nivel con círculo gra- 


\section{F. JOSE GONZALEZ GONZALEZ Y M. BERROCOSO DOMINGUEZ}

duado, cuatro objetivos colimadores, cuatro pies de cobre, cuatro anteojos de $35 \mathrm{~cm}$. de distancia focal y seis termómetros.

\section{Metro bimetálico Brunner}

Al igual que en el caso del aparato magistral, el metro estaba compuesto por dos reglas, una de platino y otra de laton, de $1 \mathrm{~m}$. de largo, 2,25 cm. de ancho y 0,5 de espesor. En los extremos de la regla de latón existían dos grapas, una de latón y otra de platino. En la segunda de ellas, iba grabada una escala compuesta por 100 divisiones separadas 2,5 decimilimetros. En los extremos de la regla de platino dos trazos señalaban la distancia de un metro a una determinada temperatura. A continuación de estos trazos, hacia el centro de la regla, estaba grabada una escala, que formaba nonio con la escala grabada en la grapa de platino. El soporte del metro bimetálico era análogo al del aparato magistral y se componía de una barra de hierro en forma de $\mathrm{T}$ invertida, en cuyo canto superior estaban afirmadas con tornillos seis piezas de latón sobre las que iba apoyada la regla.

\section{Aparatos secundarios de medir bases}

Con una disposición exactamente igual a la del aparato magistral, fueron construidos otros dos formados únicamente por una regla de cobre sustentada en madera. Su objeto más importante, era proteger al aparato principal del abuso en su utilización, con el consiguiente riesgo de producir alguna avería grave en tan sofisticado aparato.

Cuando se pensó en la adquisición de los aparatos secundarios de medir bases, existía entre los expertos un acuerdo general sobre la buena resistencia de la madera bien preparada, impregnada de aceite y barnizada, a las alteraciones higrométricas. Como consecuencia, la casa Brunner de París recibió el encargo de construir en este material las reglas de los mencionados aparatos. Sin embargo, cuando Antonio Tomaseti procedio a estudiar los resultados obtenidos en una serie de observaciones, realizadas en septiembre de $\mathbf{1 8 6 0}$, con objeto de comprobar la fiabilidad de los nuevos instrumentos, llegó a la conclusión de que era totalmente imposible preservar a la madera de las influencias ejercidas por los cambios de temperatura y de humedad. Este convencimiento le llevó a solicitar a sus superiores una autorización, que le.fue concedida, para encargar a Brunner la construcción de unas reglas adicionales de cobre que, colocadas convenientemente junto a las de madera, pudiesen dilatar independientemente. Con ello se consiguió dotar a los aparatos 
secundarios de una gran exactitud, que permitiria utilizarlos en toda clase de operaciones geodésicas e hidrográficas de precisión ${ }^{27}$.

\section{Conclusión}

La principal conclusión que podemos extraer de este pequeño trabajo es que la Marina, a pesar de la critíca situación de la primera mitad del siglo XIX, mantuvo en funcionamiento dos de las escasas instituciones científicas existentes en nuestro país durante aquellos años.

Tanto el Observatorio como la Dirección de Hidrografia consiguieron superar la dificil crisis sufrida por la Marina como consecuencia de la batalla de Trafalgar y de la pérdida de la mayor parte de los territorios de ultramar. Es cierto que no fue éste un período de grandes iniciativas y que ambas instituciones se dedicaron principalmente a intentar sobrevivir. Sin embargo, el simple hecho de haber mantenido una cierta actividad, situó a la Dirección de Hidrografía y al Observatorio en una buena posición para aprovechar el resurgir de las actividades científicas que comenzó a detectarse durante los últimos años del reinado de Isabel II. De esta forma, las iniciativas oficiales para llevar a cabo en España la triangulación geodésica de primer orden y la elaboración del Mapa Topográfico Nacional, pudieron contar con el apoyo de una Comisión Hidrográfica de la Península, organizada por la Armada, que sería la encargada de completar los trabajos citados anteriormente con el levantamiento de la cartografía de las costas españolas, y cuya labor sería completada, a su vez, por las comisiones hidrográficas de las Antillas y de las Filipinas, cuyo objetivo fue el levantamiento cartográfico de las costas de los últimos enclaves coloniales.

Los trabajos de estas comisiones tuvieron una alta calidad científica y se vieron plasmados en una innumerable cantidad de cartas y planos de las costas, fondeaderos y puertos de todo el territorio nacional. Como hemos podido comprobar, para ello fueron utilizados instrumentos científicos adquiridos al efecto, entre los que cabría destacar un aparato magistral de medir bases, instalado en el Observatorio de San Fernando, que fue construido en París por la casa Brunner, a semejanza del fabricado para la Comisión del Mapa de España.

\footnotetext{
27 Véase el expediente «Colecciones completas de instrumentos geodésicos para el levantamiento de planos (1859-1862)", Arćhivo-Museo Don Alvaro de Bazán (Viso del Marqués, Ciudad Real), serie Depósito Hidrográfico, Asuntos Particulares, Leg, 4929.
} 


\section{APENDICE I}

\section{TRABAJOS DE LA COMISION HIDROGRAFICA DE LA PENINSULA (1862-1876)}

\section{2}

Se midió, como ensayo, una base geodésica de $900 \mathrm{~m}$. en Huelva, utilizada en el levantamiento de los planos de los ríos Tinto y Odiel.

1863

Se estudió el terreno en que debía ser medida la base definitiva para ligarla al lado de la cadena Vigía de Sanlúcar-Trebujena, y se proyectó ésta hasta el vértice Meca (Cabo de Trafalgar).

\section{4}

Se terminaron los proyectos de medición de primer y segundo orden desde Portugal hasta el Cabo de Trafalgar y el plano hidrográfico de Huelva (Tinto y Odiel).

1865

Comenzaron los ensayos de la medición de primer orden, conforme fueron terminados los heliotropos encargados a Torres, instrumentario del Observatorio.

\section{6}

Se midió la triangulación proyectada desde Portugal a Trafalgar Se hizo el levantamiento de la costa comprendida entre Rota y la desembocadura del Guadalquivir. 
Se midió la base de Alventus con el aparato de tres reglas de la colección n. ${ }^{\circ} 1$ de Brunner (que ya había sido probado en Huelva). Se midieron también los ángulos de los cuadriláteros para ligarla al lado Vigía de SanlúcarTrebujena.

\section{8}

Se redujo la medición de la base de Alventus, en la hipótesis de ser $4 \mathrm{~m}$. justos la distancia que separaba los puntos de referencia del comparador Brunner instalado en el Observatorio, con el que se habían verificado las observaciones de dilatación de las reglas. Se llevó a cabo, además, la observación y cálculo del azimut astronómico del vértice Puerto desde el vértice Observatorio de San Fernando.

1869

Se levantaron varios planos de puerto y de costa. Se proyectó la triangulación hasta Tarifa.

1870

Se midió la triangulación hasta Tarifa y se proyectó hasta Málaga.

1871

Se midió la cadena de primer orden hasta el lado Pizarra-Mijas. Se eligió el sitio para medir la base de Roquetas y se estableció el teodolito astronómico en la Alcazaba de Almería.

1872

Se midió dos veces la base de Roquetas y se redujeron ambas mediciones. Se llevaron a cabo observaciones de latitud y longitud astronómicas en la Alcazaba de Almería. Se observó el azimut astronómico del vértice Punta del Río (Almería). Se proyectó y midió la cadena desde Motril a Almería. 


\section{F. JOSE GONZALEZ GONZALEZ Y M. BERROCOSO DOMINGUEZ}

1873

Se proyectó la cadena desde Pizarra-Mijas hasta Motril, midiéndose una parte de ella. También se proyectó y midió la triangulación entre Almería y Monte Cope. Se iniciaron las observaciones de comparación de las reglas geodésicas de Brunner, utilizadas para medir las bases; con la regla bimetálica magistral instalada en el Observatorio.

\section{4}

Fueron terminadas la medición de la cadena entre Pizarra-Mijas y Motril, las observaciones de comparación de las reglas y el cálculo de su reducción. Una vez conocidos los valores absolutos de aquellas; se hallaron los correspondientes a las bases de Alventus y Roquetas.

\section{5}

Debido a las diferencias constatadas entre la longitud de Almería deducida geodésicamente y la calculada directamente por métodos astronómicos; se llevó a cabo una nueva observación de la misma, además de situar astronómicamente la isla de Alborán. Se midió la cadena entre Monte Cope hasta el Cabo de San Antonio.

\section{6}

Se inició la triangulación desde el Cabo de San Antonio hasta el puerto de los Alfaques. 


\section{APENDICE II}

\section{TRABAJOS DE LA COMISION HIDROGRAFICA DE LA PENINSULA}

(1877-1887)

\section{Trabajos geodésicos}

- Memoria sobre fórmulas para el cálculo de posiciones geográficas.

- Plantillas de medición de ángulos horizontales y verticales (triangulación de $1 .^{\circ}$ y $2 .^{\circ}$ orden, de Peñíscola a Francia).

- Plantillas originales de medición de $3 .^{\text {er }}$ orden (de El Fangar a Francia).

- Extracción de ángulos horizontales y verticales de las plantillas de $1 .^{\circ}$ y $2 .^{\circ}$ orden.

- Plantillas originales de medición de ángulos horizontales y verticales (triangulación de $10^{\circ}$ y $2 .^{\circ}$ orden, de Almería a Los Alfaques).

- Plantillas originales de mediciones auxiliares de 3 er $^{\text {er }}$ orden (de Cartagena a Los Alfaques).

- Cálculo de las triangulaciones de $1 .^{\circ}, 2 .^{\circ}$ y $3 .^{\text {er }}$ orden.

- Cálculo de las elevaciones de vértices de $1 .^{\circ}$ y $2 .^{\circ}$ orden (de Almería a Los Alfaques).

- Cálculo de las elevaciones de vértices de $1 .^{\circ}$ y $2 .^{\circ}$ orden (de Vista Hermosa a Francia).

- Cálculo de las elevaciones de algunos puntos de 3 er $^{\text {er }}$ orden (de Almería a Los Alfaques).

- Cálculos de la elevaciones tomadas en los trabajos parcelarios (de Cartagena a Francia).

- Elevaciones de montes tomadas desde el mar.

- Resumen general de elevaciones (de Almería a Francia).

- Cálculo de las posiciones geográficas (de Portugal a Francia).

- Medición de la base geodésica de Ampolla.

\section{Trabajos astronómicos}

- Determinación de latitud y azimut en Valencia (1878).

- Determinación de la diferencia de longitud entre Valencia y San Fernando (1881). 
- Determinación de la diferencia de longitud entre Tarragona y San Fernando (1883).

- Determinación de latitud y azimut en Tarragona (1883).

- Determinación de la diferencia de longitud entre Almería y San Fernando (1885).

- Determinación de latitud y azimut en Rosas (1885).

- Determinación de la diferencia de longitud entre Rosas y San Fernando (1885). 